almost everywhere. This hypothesis is satisfied in all of the physical examples known so far.

In addition it generally happens that $\lambda_{1}<0$, so that the inequality $u(x) \geqq \lambda_{1} u_{0}(x)$ is vacuously true when $u(x)$ is in $U$, i.e., a true absolute minimum occurs.

\title{
BIBLIOGRAPHY
}

1. G. Goertzel, Minimum critical mass and flat flux, Journal of Reactor Science and Technology vol. 2 (1952) pp. 19-25 (declassified 11/10/52, CF-52-2-9).

2. A. C. Zaanen, On the theory of linear integral equations, III, Neder. Akad Wetensch. Amsterdam vol. 49 (1946) pp. 292-301.

Nuclear Development Associates, Inc.

\section{A CHARACTERIZATION OF ANALYTIC FUNCTIONS ${ }^{1}$}

J. E. MCLAUGHLIN AND C. J. TITUS

1. Let $f$ be a mapping of an open set $D$ in the $x y$-plane into the $u v$-plane where the component functions $u$ and $v$ are continuously differentiable. If the mapping is sense preserving, then the Jacobian, $J(f)$, cannot be negative at any point. If, by analogy with analytic functions, one assumes also that the Jacobian is zero only if the Jacobian matrix has rank zero, then one is led to the study of a family of mappings $\mathfrak{F}$ where

$$
\begin{gathered}
f \in \mathfrak{F} \rightarrow J(f) \geqq 0, \\
J(f)=0 \rightarrow \text { rank of } J \text { is zero. }
\end{gathered}
$$

The purpose of this paper is in part to show that if any real linear vector space of mappings $\mathfrak{B}$ is contained in $\mathfrak{F}$ and $\mathfrak{B}$ contains a pair of analytic functions whose derivatives are independent on $D$, then $\mathfrak{B}$ contains only analytic functions.

We first prove an algebraic lemma upon which the whole characterization rests.

2. Let $\mathbb{S}$ be the vector space of all $2 \times 2$ matrices of the form

$$
\left(\begin{array}{rr}
a & -b \\
b & a
\end{array}\right)
$$

Received by the editors October 28, 1953.

1 The work in this paper was sponsored in part by Office of Naval Research. 
where $a$ and $b$ are real numbers. Then $\mathbb{S}$ is isomorphic to the complex numbers and $\mathbb{S}$ enjoys the property

$$
\begin{aligned}
C & \in \mathbb{E} \rightarrow|C| \geqq 0, \quad|C|=\operatorname{det} C, \\
|C| & =0 \rightarrow C=0 .
\end{aligned}
$$

We first prove

LEMMA 1. C5 is a maximal vector space having property $(\mathrm{P})$ in the algebra of all $2 \times 2$ matrices.

If the lemma is false, there is a vector space $\mathfrak{B}$ with property $(\mathrm{P})$ which properly includes $\mathbb{E}$. Let $D \in \mathfrak{B}, D \in \mathbb{C}$. Then the matrices $I, J$, and $D$ are linearly independent. ( $I$ is the identity, $J^{2}=-I$.) It then follows that $I, J, D$, and $D J$ are linearly independent. For suppose $\lambda, \mu, \sigma, \tau$, not all zero, exist so that

$$
\lambda I+\mu J+\sigma D+\tau D J=0 .
$$

Then right multiplication by $J$ gives

$$
\lambda J-\mu I+\sigma D J-\tau D=0 .
$$

Since $I, J$, and $D$ are independent, it follows from (2) that $\tau \neq 0$ and from (3) that $\sigma \neq 0$. Thus

$$
D J=-\left(\frac{\lambda}{\tau} I+\frac{\mu}{\tau} J+\frac{\sigma}{\tau} D\right)=\frac{\mu}{\sigma} I-\frac{\lambda}{\sigma} J+\frac{\tau}{\sigma} D
$$

which implies $-\sigma / \tau=\tau / \sigma$ or $\sigma^{2}+\tau^{2}=0$. This is a contradiction and the independence of $I, J, D$, and $D J$ is established.

For any real numbers $\lambda, \mu, \sigma, \tau$, with $\sigma^{2}+\tau^{2} \neq 0$,

$$
\begin{aligned}
|\lambda I+\mu J+\sigma D+\tau D J|= & |\lambda I+\mu J+D(\sigma I+\tau J)| \\
= & \left|(\lambda I+\mu J)(\sigma I+\tau J)^{-1}+D\right| \\
& \cdot|\sigma I+\tau J|>0,
\end{aligned}
$$

since the matrix in each factor is in $\mathfrak{B}$ and is not zero. Hence the space spanned by $I, J, D$, and $D J$, which is just the space of all $2 \times 2$ matrices, has property $(\mathrm{P})$ and this contradiction establishes Lemma 1.

LеммA 2. Let $\mathfrak{W}$ be any vector space in the space of all real $2 \times 2$ matrices which enjoys the property (P). Then $\mathfrak{B}$ is either one-dimensional and equivalent to the scalar matrices or $\mathfrak{B}$ is two-dimensional and equivalent to the space 5 .

The one-dimensional case is completely trivial. Suppose $\mathfrak{W}$ has 
dimension greater than one. Then there exists a pair of matrices $U, V$ in $\mathfrak{W}$ which are linearly independent. By property $(\mathrm{P})$ one has for all real $\lambda$ and $\mu$ that

$$
|\lambda U+\mu V| \geqq 0
$$

which implies

$$
\left|\lambda I+\mu V U^{-1}\right| \geqq 0,
$$

and equality holds if and only if $\lambda=\mu=0$.

Let $V U^{-1}=A$, and let $\widehat{\mathfrak{B}}$ denote the two-dimensional space spanned by $I$ and $A$. From (4) we see that $\widehat{\mathfrak{B}}$ also has property (P). Since the minimum function of $A$ is quadratic, it is clear that $\widehat{\mathfrak{W}}$ is closed under multiplication. Similarly one sees that if $\lambda I+\mu A \neq 0$, then $(\lambda I+\mu A)^{-1} \in \widehat{\mathfrak{W}}$. Hence $\widehat{\mathfrak{W}}$ is a two-dimensional division algebra over the reals. Then $\widehat{\mathfrak{W}}$ is isomorphic to the complex numbers and therefore by the Skolem-Noether theorem [1] there exists a nonsingular $P$ such that $\widehat{\mathfrak{W}}=P\left(\mathcal{S}^{-1}\right.$. Then $V U^{-1}=P C P^{-1}$ for some $C \in \mathfrak{S}$. If we set $P^{-1} U=Q$, we have $U=P I Q, V=P C Q$. If $\mathfrak{B}$ contains a matrix $W$ such that $U, V$, and $W$ are linearly independent, then the space generated by $I, C$, and $P^{-1} W Q^{-1}$ would be three-dimensional, contain $(\mathcal{S}$, and enjoy property (P). This contradicts Lemma 1 and hence $\mathfrak{B}$ is two-dimensional and equivalent to $\mathfrak{S}$.

Let $\mathfrak{F}$ be a family of mappings defined on a domain $D$ which satisfy the following conditions:

(a) $f_{1}, f_{2} \in \mathfrak{F} \rightarrow \lambda_{1} f_{1}+\lambda_{2} f_{2} \in \mathfrak{F}$ for all real $\lambda_{1}$ and $\lambda_{2}$.

(b) $f \in \mathfrak{F} \rightarrow f$ is continuously differentiable.

(c) $f \in \mathfrak{F} \rightarrow$ the Jacobian of $f$ is non-negative and zero only if it is of rank zero.

THEOREM. If $\mathfrak{F}$ contains a pair of analytic functions, $f_{1}$ and $f_{2}$, whose complex derivatives are independent on $D\left(\operatorname{Im} \bar{f}_{1}^{\prime} f_{2}^{\prime} \neq 0\right.$ on $\left.D\right)$, then $\mathfrak{F}$ is a linear vector space of analytic functions.

Proof. Let $\Im$ denote the set of all Jacobian matrices of mappings in $\mathfrak{F}$. They must form at every point $\left(x_{0}, y_{0}\right)$ of $D$ a linear vector space of $2 \times 2$ matrices with the property $(\mathrm{P})$. Thus the Jacobian matrix $J$ for an arbitrary mapping $f \in \mathfrak{F}$ at $\left(x_{0}, y_{0}\right)$ must have the form

$$
\left(\begin{array}{ll}
u_{x} & u_{y} \\
v_{x} & v_{y}
\end{array}\right)_{\left(x_{0}, y_{0}\right)}=P C Q \quad \text { where } C \in \mathbb{E} .
$$

Since $f_{1}$ and $f_{2}$ are analytic functions and their derivatives are independent, their Jacobian matrices at $\left(x_{0}, y_{0}\right)$ have the form 


$$
J\left(f_{1}\right)=\left(\begin{array}{cc}
\lambda_{1} & -\mu_{1} \\
\mu_{1} & \lambda_{1}
\end{array}\right)=C_{1}, \quad J\left(f_{2}\right)=\left(\begin{array}{rr}
\lambda_{2} & -\mu_{2} \\
\mu_{2} & \lambda_{2}
\end{array}\right)=C_{2}
$$

where $C_{1}$ and $C_{2}$ are independent. There exist then $\widehat{C}_{1}$ and $\widehat{C}_{2}$ in $\sqrt{ }$ such that

$$
P \widehat{C}_{1} Q=C_{1}, \quad P \widehat{C}_{2} Q=C_{2} .
$$

However, since a vector space with the propcrty $(\mathrm{P})$ can be at most two-dimensional one has that $P \mathbb{S} Q=\mathbb{C}$. Thus our given Jacobian matrix at $\left(x_{0}, y_{0}\right)$ must be in $(5)$ which implies that the component functions of $f$ satisfy the Cauchy-Riemann equations. Since $\left(x_{0}, y_{0}\right)$ was arbitrary and the Jacobian was assumed to be continuous, it follows that $f$ is an analytic function as was to be proved.

\section{BIBLIOGRAPHY}

1. Hermann Weyl, The classical groups, Princeton University Press, 1946, p. 282, Theorem (9.1.D).

UNIVERSITY OF MiChIGAN 